04

\title{
Управление обтеканием круглого цилиндра с помощью коронного разряда
}

\author{
(C) М.Е. Ренев, Ю.Ф. Сафронова,ף Ю.К. Стишков
}

Санкт-Петербургский государственный университет, 199034 Санкт-Петербург, Россия

ฯ e-mail: y.safronova@spbu.ru

Поступило в Редакцию 11 января 2019 г.

В окончательной редакции 11 января 2019 г.

Принято к публикации 25 марта 2019 г.

Исследовано воздействие электрического ветра, вызванного положительным коронным разрядом, на обтекание кругового цилиндра потоком воздуха с числами Рейнольдса 2400, 3200. Геометрия электродов цилиндр-провод была рассмотрена для двух положений коронирующего электрода относительно цилиндра: за цилиндром и двух симметричных проводов сверху и снизу цилиндра. Проведено прямое численное моделирование электрогидродинамической задачи, использовалась оригинальная униполярная модель коронного разряда. Рассмотрено влияние тонкой струйки электрического ветра, направленной от коронирующего электрода к цилиндру, на структуру вихревого следа за цилиндром и силу лобового сопротивления. Показано, что при расположении двух коронирующих электродов сверху и снизу цилиндра электрический ветер препятствует образованию дорожки Кармана, а также существенно уменьшает аэродинамическое сопротивление цилиндра. При расположении коронирующего электрода за цилиндром коронный разряд и электрический ветер приводят к формированию и развитию крупных вихрей в следе за цилиндром, что приводит к существенным колебаниям аэродинамического сопротивления цилиндра. Было показано, что коронный разряд значительно изменяет характеристики дорожки Кармана: с увеличением напряжения до $30 \mathrm{kV}$ частота отрыва вихрей уменьшается в 2.5 раза, размеры вихрей и скорость их вращения заметно возрастают. Сила лобового сопротивления имеет квазипериодический характер, ее средняя величина линейно зависит от напряжения короны.

Ключевые слова: положительный коронный разряд, электрический (ионный) ветер, ЭГД течение, дорожка Кармана, управление отрывом пограничного слоя, коэффициент лобового сопротивления.

DOI: 10.21883/JTF.2019.09.48060.5-19

\section{Введение}

Управление обтеканием с помощью коронного разряда относится к активным электрогидродинамическим методам, в нем используется энергия источника электрического тока. Этот способ привлекает внимание простотой, надежностью, малыми размерами конструкций, которые не включают в себя движущиеся части и экономно расходуют энергию. К тому же в отличие от пассивных методов, в этом случае не нужно изменять геометрию поверхности обтекаемого тела. Кроме того, величину воздействия можно легко регулировать изменением напряжения. Постоянная времени для коронного разряда составляет доли микросекунд, что на порядки больше установления гидродинамических процессов. Это открывает возможности для разработки методов управления с обратной связью. Принципиальная возможность управления течением и аэродинамическими характеристиками обтекаемых тел в результате действия электрических сил была показана многими экспериментальными и теоретическими работами, например, [1-4], однако этот вопрос требует дальнейшего изучения. Большое количество работ посвящено управлению аэродинамикой крыла с помощью электрического разряда, в то время как обтекания цилиндра касаются лишь несколько. Среди них подавляющее большинство - экспериментальные работы. Цилиндр - универсальная форма, применимая для многих приложений и хорошо изученная экспериментально, поэтому, по мнению авторов, исследование влияния тонкой, около миллиметра в сечении, струйки электрического ветра (ЭВ), создаваемого коронным разрядом, на обтекание цилиндра представляет не только практический, но и теоретический интерес.

При поперечном обтекании цилиндра вязкой средой в диапазоне чисел Рейнольдса $\mathrm{Re}=40-10^{5}$ от поверхности тела периодически отрываются вихри, и за цилиндром образуется след, называемый дорожкой Кармана [5]. Механические колебания, вызванные срывом вихрей, могут приводить к вибрации, акустическому шуму и при совпадении частоты отрыва вихрей с собственной частотой конструкции к ее разрушению. Поэтому среди задач регулировки обтеканием цилиндрических тел, наряду с уменьшением силы лобового сопротивления, можно выделить изменение амплитуды и частоты колебания действующих на тело сил. Это связано с изменением параметров дорожки Кармана и переходом нестационарного режима обтекания в стационарный.

Для тел с плохообтекаемой формой, таких как цилиндр, сопротивление вязкого трения мало по сравнению с сопротивлением давления, следовательно, умень- 
шение лобового сопротивления сводится к уменьшению разности давлений в носовой и кормовой части тела. Эту задачу можно решать смещением точки отрыва пограничного слоя. Можно выделить два пути решения: сила со стороны электрического разряда действует вдоль поверхности тела, ускоряя частицы воздуха пограничного слоя, или перпендикулярно движению газа, присоединяя оторвавшийся пограничный слой. Авторы экспериментальной работы [3] рассматривали первый путь решения. Для этого высоковольтный электродпроволока ставился в носовой точке стагнации цилиндра, а заземленный электрод - в кормовой области. Для относительно низких чисел Рейнольдса 2500 (скорость набегающего потока $0.7 \mathrm{~m} / \mathrm{s}$ ) визуализация потока при помощи струек дыма показала, что включение напряжения изменило вид вихревой дорожки за цилиндром: точки отрыва пограничного слоя сместились вниз по течению, дорожка теряла свою четкую структуру, крупные вихри не наблюдались. PIV-измерения показали, что создается ускорение в области отрыва пограничного слоя. Однако такая геометрия не перспективна для задачи уменьшения лобового сопротивления.

В работе [4] в аэродинамической трубе проводилось экспериментальное изучение влияния коронного разряда на обтекание цилиндра для разных вариантов расположения коронирующих электродов. Скорость набегающего потока изменялась от 1 до $2 \mathrm{~m} / \mathrm{s}$, так, что число Рейнольдса находилось в диапазоне $4000<\mathrm{Re}<8000$. Роль коронирующего электрода выполняли один или два провода, симметрично расположенных относительно плоскости симметрии. Положение проводов характеризовалось углами $\theta=45,90,135$ и $180^{\circ}$. Визуализация течения с помощью дымового провода показала, что в зависимости от положения высоковольтных электродов точка отрыва пограничного слоя смещается вверх или вниз по течению, увеличивая или уменьшая размер следа. Также проводилось измерение давления на поверхности цилиндра, которое подтвердило возможность регулирования силы сопротивления положением электродов и величиной напряжения коронного разряда. С точки зрения уменьшения лобового сопротивления можно считать наиболее перспективными положения проводов для $\theta=90^{\circ}$ и $180^{\circ}$.

Работа [6] посвящена численному моделированию ЭГД течения для системы электродов $\theta=90^{\circ}$ из [4]. Рассматривалась модель турбулентного течения с числами Рейнольдса $4000<\operatorname{Re}<16000$. Авторы подтверждают, что, согласно экспериментальным результатам [4], при включении коронного разряда размер турбулентного следа за цилиндром уменьшается.

В настоящей работе авторы сосредоточились на особенностях управления характеристиками дорожки Кармана при обтекании цилиндра с помощью коронного разряда для относительно низких чисел Рейнольдса 2400 и 3200. Несмотря на то что такие числа Рейнольдса соответствуют переходному режиму, след характеризуется явно выраженными крупными вихрями с четкой структурой, что позволяет разрешить его методом прямого численного моделирования. Рассмотрены два варианта размещения коронирующих электродов. В первом случае высоковольтные электроды располагались симметрично над и под цилиндром, создавая ЭВ, перпендикулярный основному потоку. Это заставляло присоединиться оторвавшийся пограничный слой, уменьшало размеры следа и для некоторых напряжений приводило нестационарное течение к стационарному. Во втором случае высоковольтный электрод располагался за цилиндром, ЭВ был направлен противоположно основному потоку, увеличивая размеры следа, что приводило к большей нестационарности потока. Задача решалась с помощью численного моделирования.

\section{Модель коронного разряда}

Коронный разряд возникает в системах электродов с малым радиусом кривизны, вблизи которых локализована высокая напряженность электрического поля. Высокая напряженность приводит к ударной ионизации, лавинообразно повышая число заряженных частиц около электрода. Узкая (около $0.1 \mathrm{~mm}$ ) область вблизи высоковольтного электрода, где происходят ионизационные процессы и взаимодействуют заряженные частицы нескольких сортов: различные ионы и электроны, называется чехлом коронного разряда. Во внешней зоне, вне чехла коронного разряда, находятся ионы только одного знака, которые дрейфуют к противоположному электроду под действием электрического поля. При столкновении с нейтральными молекулами воздуха дрейфующие ионы передают им свой импульс, вовлекая в движение и, таким образом, создавая ЭВ.

В случае положительного высоковольтного электрода (положительной короны) электроны образуются в небольшой окрестности чехла за счет фотоионизации [7], втягиваются в чехол, лавинообразно размножаются и поглощаются высоковольтным электродом. Во внешнюю зону в этом случае направлен поток положительных ионов из чехла короны. Малые по сравнению с межэлектродным расстоянием размеры чехла позволяют использовать упрощенную (так называемую униполярную) математическую модель коронного разряда. В униполярной модели рассматривается только внешняя зона разряда, в которой дрейфуют заряженные частицы одного знака, а процессы ионизации в чехле заменяются граничным условием на высоковольтном электроде. Как правило, это условие выбирается на основании эмпирически установленных закономерностей и поэтому традиционные модели требуют переопределения коэффициентов при любом изменении геометрии. В настоящей работе используется оригинальное граничное условие на высоковольтном электроде в виде дифференциального уравнения, задающего скорость изменения потока ионов из чехла коронного разряда $[8,9]$. Входные параметры 
модели содержат физические величины, поддающиеся независимому измерению, такие как коэффициент ионизации, подвижность ионов, коэффициент диффузии ионов, критическое число ионизационных столкновений, необходимое для зажигания разряда. Модель тестировалась на ряде задач с ЭВ для разных конфигураций электродов и дала хорошее совпадение с экспериментом.

Униполярная модель коронного разряда описывается системой (1)-(4). (1) - уравнение Пуассона для электрического потенциала $\varphi$ и уравнение связи вектора напряженности электрического поля $\mathbf{E}$ и потенциала, где $|e|-$ абсолютное значение заряда электрона $1.6 \cdot 10^{-19} \mathrm{C}, \varepsilon_{0}-$ диэлектрическая постоянная $8.85 \cdot 10^{-12} \mathrm{~F} / \mathrm{m}, n-$ концентрация положительных ионов во внешней зоне. Уравнение Нернста-Планка (2) не содержит функции источников и учитывает изменение концентрации ионов за счет диффузии с эффективным коэффициентом диффузии $D=10 \mathrm{~mm}^{2} / \mathrm{s}$ и за счет миграции в электрическом поле с эффективной подвижностью положительных ионов $b=2.1 \cdot 10^{-4} \mathrm{~m}^{2} /(\mathrm{V} \cdot \mathrm{s})$.

Рождение положительных ионов в чехле коронного разряда описывается граничным условием на коронирующем электроде в виде скорости изменения плотности потока положительных ионов $\partial j_{0} / \partial t$ во внешнюю зону (3). Коэффициент $\exp (-M)$ характеризует интенсивность затравочных электронов за счет фотоионизации; $M$ - число ионизационных столкновений, достаточное для зажигания коронного разряда; $\tau$ время пролета положительного иона от границы чехла коронного разряда до коронирующего электрода $0.1 \mathrm{~ms}$; $m$ - число ионизирующих столкновений вдоль силовой линии в чехле коронного разряда, определяемое соотношением (4), где $\alpha(E)$ - эффективный коэффициент ионизации, который рассматривается как функция от электрического поля [7], $s$ - координата вдоль силовой линии.

$$
\begin{gathered}
\Delta \varphi=-\frac{|e| n}{\varepsilon_{0}}, \mathbf{E}=-\nabla \varphi, \\
\frac{\partial n}{\partial t}+\nabla \cdot(-D \nabla n+b n \mathbf{E})=0, \\
\frac{\partial j_{0}}{\partial t}=j_{0} \frac{\exp (-M) \exp (m)-1}{\tau}, \\
m=\int_{0}^{s} \alpha(E) d s .
\end{gathered}
$$

\section{Моделирование ЭГД течения}

Система электродов цилиндр-провод находится в аэродинамической трубе со скоростью набегающего потока воздуха $V_{0}$. Цилиндр радиусом $R=3 \mathrm{~cm}$ заземлен. К проводу радиусом $r=45 \mu \mathrm{m}$ приложено высокое положительное напряжение $U$. Провод помещен на расстоянии $h=R$ от поверхности цилиндра. Рассматривались два варианта расположения высоковольтных электродов

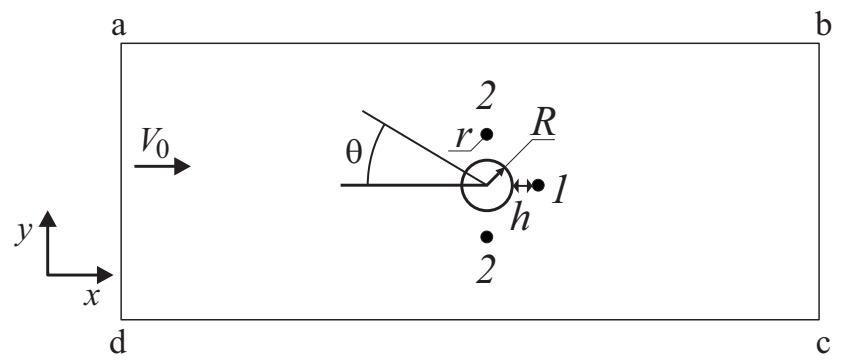

Рис. 1. Геометрия модели: 1,2 - два варианта расположения высоковольтных электродов относительно цилиндра.

относительно цилиндра: 1) одиночный провод находился за цилиндром $\left(\theta=180^{\circ}\right.$ на рис. 1$\left.) ; 2\right)$ два провода располагались симметрично сверху и снизу цилиндра $\left(\theta= \pm 90^{\circ}\right.$ на рис. 1). Размеры установки совпадают с рассматриваемыми в экспериментальной работе [4].

Моделирование проводилось для плоской геометрии. Область моделирования показана на рис. 1, размеры проводов изображены не в масштабе. Длина трубы $a b=100 \mathrm{~cm}$, высота $a d=40 \mathrm{~cm}$. Рассматривались скорости $V_{0}$, равные 0.6 и $0.8 \mathrm{~m} / \mathrm{s}$. При таких скоростях обдува цилиндра с выключенным коронным разрядом число Рейнольдса, рассчитанное по формуле (5), было равно 2400 и 3200 соответственно. Для проволоки число Рейнольдса не превышало 5:

$$
\operatorname{Re}=\frac{\rho V_{0} 2 R}{\mu}
$$

где $\rho$ - плотность воздуха $1.2 \mathrm{~kg} / \mathrm{m}^{3}, \mu$ - динамическая вязкость воздуха $18 \mu \mathrm{Pa} \cdot \mathrm{s}$. Течение описывалось системой уравнений Навье-Стокса для несжимаемой жидкости с объемной электрической силой (6):

$$
\rho\left(\frac{\partial \mathbf{V}}{\partial t}+(\mathbf{V} \cdot \nabla) \mathbf{V}\right)=-\nabla \rho+\mu \Delta \mathbf{V}+|e| n \mathbf{E}, \nabla \cdot \mathbf{V}=0
$$

где $\mathbf{V}$ - вектор скорости среды, $p$ - относительное давление.

Моделирование системы уравнений (1)-(4) и (6) проводилось методом конечных элементов в пакете Comsol Multiphysics. Граничные условия для моделирования показаны в таблице. Для гидродинамической задачи на стенках $a b$ и $c d$, на поверхности цилиндра и провода задавалось условие прилипания. На входе $\mathrm{ad}$ задавалась скорость, перпендикулярная границе (n вектор нормали к границе). Относительное давление $p_{0}$ на границе $b c$ при выходе в атмосферу задавалось, равным нулю. Для электростатики на внешних границах модели abcd задавалось условие не проникновения электрического поля, цилиндр был заземлен, на проводе задавался высокий положительный потенциал. Для уравнения переноса частиц на внешних границах модели задавалось условие не проникновения положительных ионов (j - плотность потока ионов), на цилиндре 
Граничные условия

\begin{tabular}{l|c|c|c}
\hline & Гидродинамика (6) & Электростатика $(1)$ & Перенос частиц (2) \\
\hline Стенки $\mathrm{ab}, \mathrm{cd}$ & $\mathbf{V}=0$ & $(\mathbf{n}, \mathbf{E})=0$ & $(\mathbf{n}, \mathbf{j})=0$ \\
Вход ad & $\mathbf{V}=-\mathbf{n} V_{0}$ & $(\mathbf{n}, \mathbf{E})=0$ & $(\mathbf{\mathbf { n }})=0$ \\
Выход bc & $p_{0}=0$ & $(\mathbf{n}, \mathbf{E})=0$ & $(\mathbf{n}, \mathbf{j})=0$ \\
Цилиндр & $\mathbf{V}=0$ & $\varphi=0$ & $\mathbf{j}_{g}=-(\mathbf{n}, \mathbf{j})$ \\
Провод & $\mathbf{V}=0$ & $\varphi=+U$ & $\mathbf{j}_{c}=\mathbf{n} j_{0}, j_{0}$ из $(3)$
\end{tabular}

задавалось свободное прохождение ионов, на проводе условие рождения ионов ( $j_{0}$ рассчитывалось из дифференциального уравнения (3)). В начальный момент времени напряжение на проводе и скорость на входе были равны нулю, далее они линейно возрастали до заданного значения. В настоящей работе анализируется квазистационарное решение.

Для конечно-элементной модели строилась свободная сетка из треугольных элементов и погранслойная сетка со сгущением вблизи границ жидкости и твердого тела, также сетка сгущалась в области вихревого следа. Анализ сеточной сходимости показал, что достаточно рассматривать около 17000 элементов.

\section{Результаты и их обсуждение}

Для рассматриваемой геометрии электродов была рассчитана вольт-амперная характеристика (BAX) коронного разряда. Как известно [7], коронный разряд имеет пороговый характер, он зажигается при некотором напряжении $U_{0}$ и далее при увеличении напряжения $U$ BAX коронного разряда растет квадратично и описывается уравнением

$$
I=K U\left(U-U_{0}\right)
$$

где $I$ - полный ток разряда. Коэффициент $K$ определяет крутизну ВАХ и зависит от геометрии электродов и подвижности положительных ионов. Подвижность ионов бралась равной $2.1 \cdot 10^{-4} \mathrm{~m}^{2} /(\mathrm{V} \cdot \mathrm{s})$. Это значение входит в диапазон используемых в литературе величин для эффективной подвижности положительных ионов воздуха [7] и хорошо согласуется с экспериментальными данными [4]. Для случая двух проводов в рассматриваемой геометрии корона на одном электроде горит практически независимо от другого. Это подтверждается тем, что для системы электродов с одним высоковольтным проводом ВАХ примерно в два раза ниже, чем для системы электродов с двумя проводами. Напряжение зажигания короны $U_{0}$ определяется величиной $M-$ числом ионизационных столкновений, достаточным для зажигания коронного разряда. В модели число $M=11$, что соответствует $U_{0}=7 \mathrm{kV}$. Эксперимент показывает [4], что в такой системе электродов пробой возникает при напряжении около $31.5 \mathrm{kV}$.
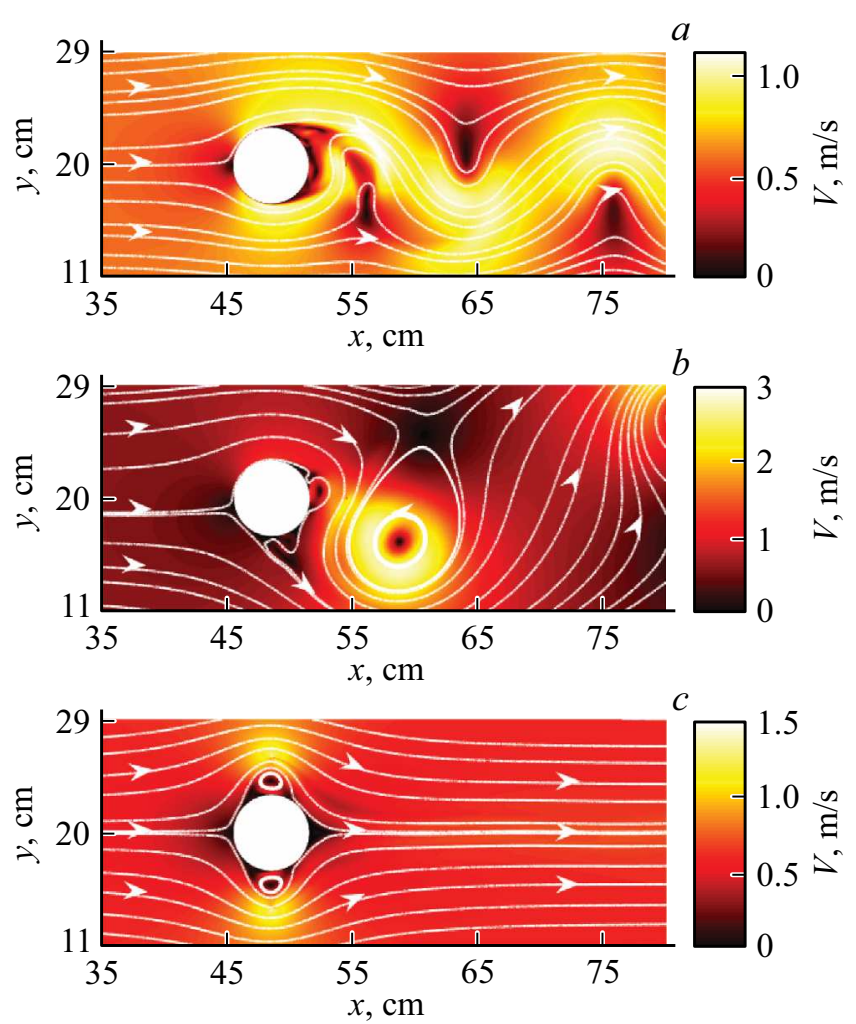

Рис. 2. Распределение модуля скорости $V$ и линии тока для $V_{0}=0.6 \mathrm{~m} / \mathrm{s}: a-U=0, b-U=21.2 \mathrm{kV}, \theta=180^{\circ}$, $c-U=16.2 \mathrm{kV}, \theta=90^{\circ}$.

Для рассматриваемых чисел Рейнольдса обтекание цилиндра потоком воздуха представляет собой квазипериодический процесс, характер которого может меняться в зависимости от расположения электродов и величины напряжения. Рис. 2 иллюстрирует это утверждение. На рисунке приведены распределения скорости вблизи цилиндра при скорости набегающего потока $V_{0}=0.6 \mathrm{~m} / \mathrm{s}$ для трех вариантов: $a-$ классическое обтекание без коронного разряда, $b$ - коронирующий электрод сзади $\left(\theta=180^{\circ}\right)$ при напряжении $21.2 \mathrm{kV}$ и $c$ - два коронирующих электрода сверху и снизу $\left(\theta=90^{\circ}\right)$ при напряжении $16.2 \mathrm{kV}$. В первом случае наблюдается классическая дорожка Кармана, течение периодически отрывается с боковой поверхности цилиндра, в следе после цилиндра в шахматном порядке наблюдаются вихри. Во втором случае из области ко- 

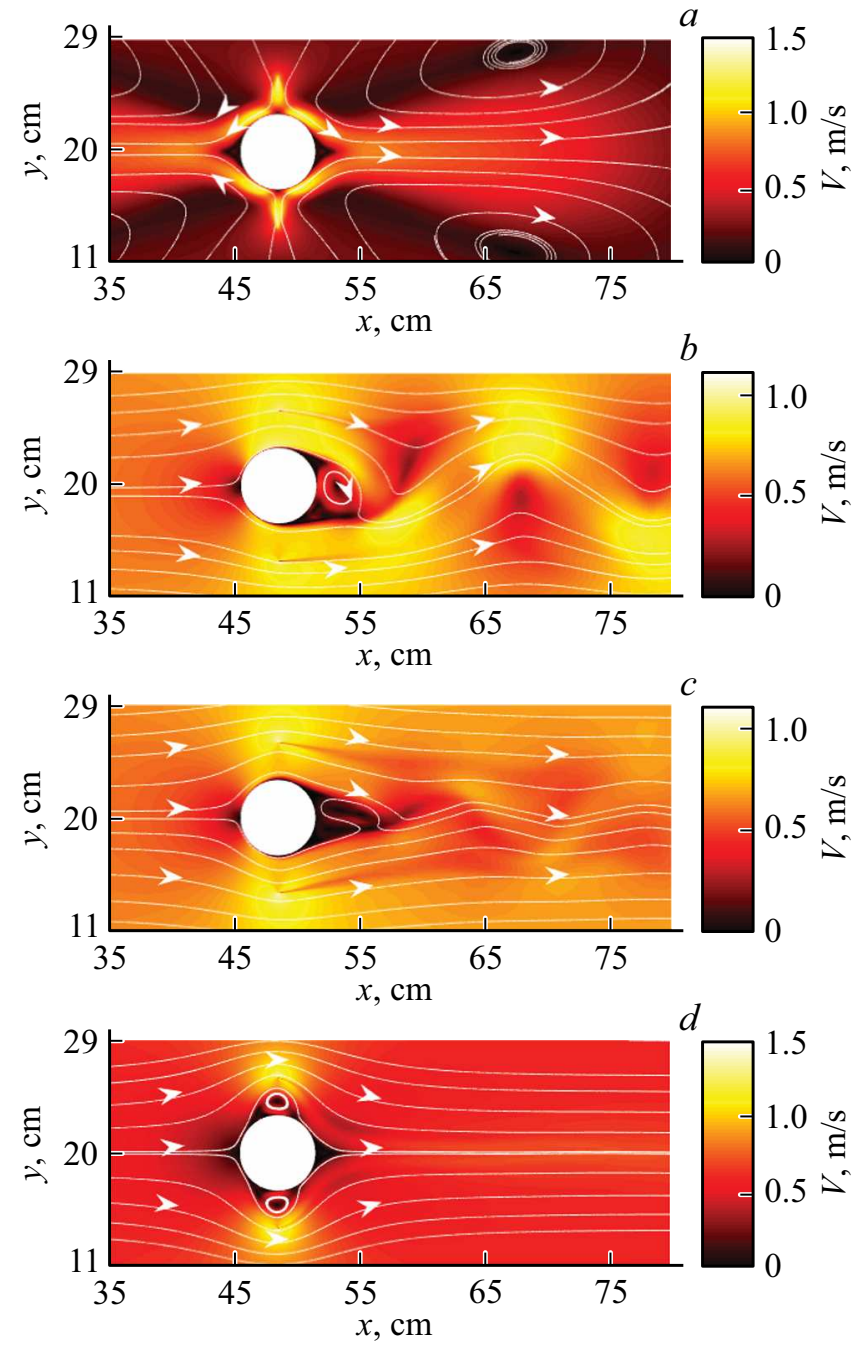

Рис. 3. Распределение модуля скорости $V$ и линии тока для $\theta=90^{\circ}: a-V_{0}=0, U=16.2 \mathrm{kV}, b-V_{0}=0.6 \mathrm{~m} / \mathrm{s}$, $U=10.2 \mathrm{kV}, c-V_{0}=0.6 \mathrm{~m} / \mathrm{s}, U=12.2 \mathrm{kV}, d-V_{0}=0.6 \mathrm{~m} / \mathrm{s}$, $U=16.2 \mathrm{kV}$.

ронирующего электрода к цилиндру дует тонкая струйка ЭВ, действующая на заднюю поверхность цилиндра и снижающая силу аэродинамического сопротивления. Однако в результате взаимодействия тонкой струйки ЭВ и набегающего потока, за цилиндром формируется выраженное кольцевое течение с повышенной скоростью вращения. Вихри приобретают большие размеры, периодически отрываются от поверхности цилиндра и сносятся вниз по течению. В третьем случае около коронирующих электродов возникает область разряжения, которая всасывает набегающий поток воздуха, смещая точку отрыва вверх по течению, причем линии тока за цилиндром практически зеркально симметричны линиям тока перед цилиндром, а область пониженного давления за цилиндром исчезла. В верхней и нижней частях цилиндра в промежутке между электродом и цилиндром образуются два мелкомасштабных вихря, отрывных пузыря, вихревого следа за цилиндром не возникает.
Рассмотрим подробнее структуру результирующего течения за цилиндром для различных положений коронирующего электрода. На рис. 3 приведены результаты расчета (линии тока и поле скоростей) течения вблизи цилиндра для случая двух коронирующих электродов $\theta= \pm 90^{\circ}$. При отсутствии внешнего потока (рис. 3, $a$ ) наблюдается характерная картина ЭВ: от коронирующих электродов распространяется тонкая центральная струйка, нормальная к поверхности цилиндра. Поперечное сечение струйки составляет 1-2 mm, максимальная скорость в струйке характерна для систем с коронным разрядом и составляет $1.5 \mathrm{~m} / \mathrm{s}$. Эта струйка, соударяясь с цилиндром, создает зону повышенного давления около его поверхности. С включением внешнего обдува наблюдается снос струйки ЭВ по течению и формирование за цилиндром вихревого следа. На рис. $3, b-d$ скорость набегающего потока постоянна и равна $0.6 \mathrm{~m} / \mathrm{s}$, а напряжение увеличивается с 10.2 до $16.2 \mathrm{kV}$. При увеличении напряжения размеры формирующихся вихрей уменьшаются, и ширина вихревого следа также уменьшается. При $U=16.2 \mathrm{kV}$ вихри в межэлектродном промежутке перестают отрываться внешним течением, и вихревой след за течением исчезает совсем. Течение становится устойчивым, форма линий тока не изменяется со временем. Линии тока основного течения втягиваются в область пониженного давления у коронирующих электродов, огибают вихри в межэлектродном промежутке и ниже по течению вновь приближаются к поверхности цилиндра. Течение напоминает ползучее, разность давлений на переднюю и заднюю стенки цилиндра стремится к нулю. При дальнейшем увеличении скорости внешнего потока вихри в межэлектродном промежутке вновь смещаются в кормовую область цилиндра, образуя симметричные зоны рециркуляции. При дальнейшем увеличении скорости симметрия нарушается, течение становится неустойчивым и вновь образуется вихревая дорожка.

На рис. 4. показаны скорости и линии тока для расположения коронирующего электрода за цилиндром. Как видно из рис. 4, $a$, при отсутствии внешнего потока ЭВ образует тонкую струйку, направленную справа налево в сторону цилиндра. При подаче внешнего потока ЭВ, направленный навстречу внешнему потоку, способствует формированию более крупных вихрей дорожки Кармана. Зарождающийся вихрь под действием тонкой струйки ЭВ прижимается к цилиндру. ЭВ, не давая вихрю оторваться, одновременно раскручивает его и способствует накоплению в нем кинетической энергии. При этом период отрыва под влиянием ЭВ существенно увеличивается. На рис. 2, $b$ заметно сильное изменение размеров вихрей и максимальных скоростей в вихре по сравнению с исходной дорожкой Кармана (рис. 2,a). Максимальный радиус вихря, наблюдаемый в работе, в два раза превышал радиус цилиндра, максимальная скорость вращения вихря возросла в три раза.

На рис. 5 приведена одна из спиралевидных траекторий пробного объема газа в вихревом следе (положение 
коронирующего электрода указано стрелкой), а также изменение вдоль этой траектории скорости, кулоновских сил и сил давления, действующих на пробный объем газа при его движении в вихревом следе. При периодическом приближении пробного объема к коронирующему электроду (на графиках эти моменты времени обозначены $t_{1}-t_{5}$ ) действующая на объем сила Кулона резко возрастает, придавая ему ускорение. Из графика рис. $5, b$ видно, что после прохождения электрода скорость вращения вихря испытывает импульсный рост под действием кулоновских сил и сил давления. При этом средняя скорость вращения увеличивается более чем в два раза, а затем после отхода вихря от электрода плавно уменьшается. Увеличенный под действием ЭВ вихрь является примером локального воздействия коронного разряда: благодаря тому, что ЭВ представляет собой тонкую струю, ускоряющую вихрь только с одной стороны, в итоге получается вихревое течение крупных размеров и возросшей скорости вращения.
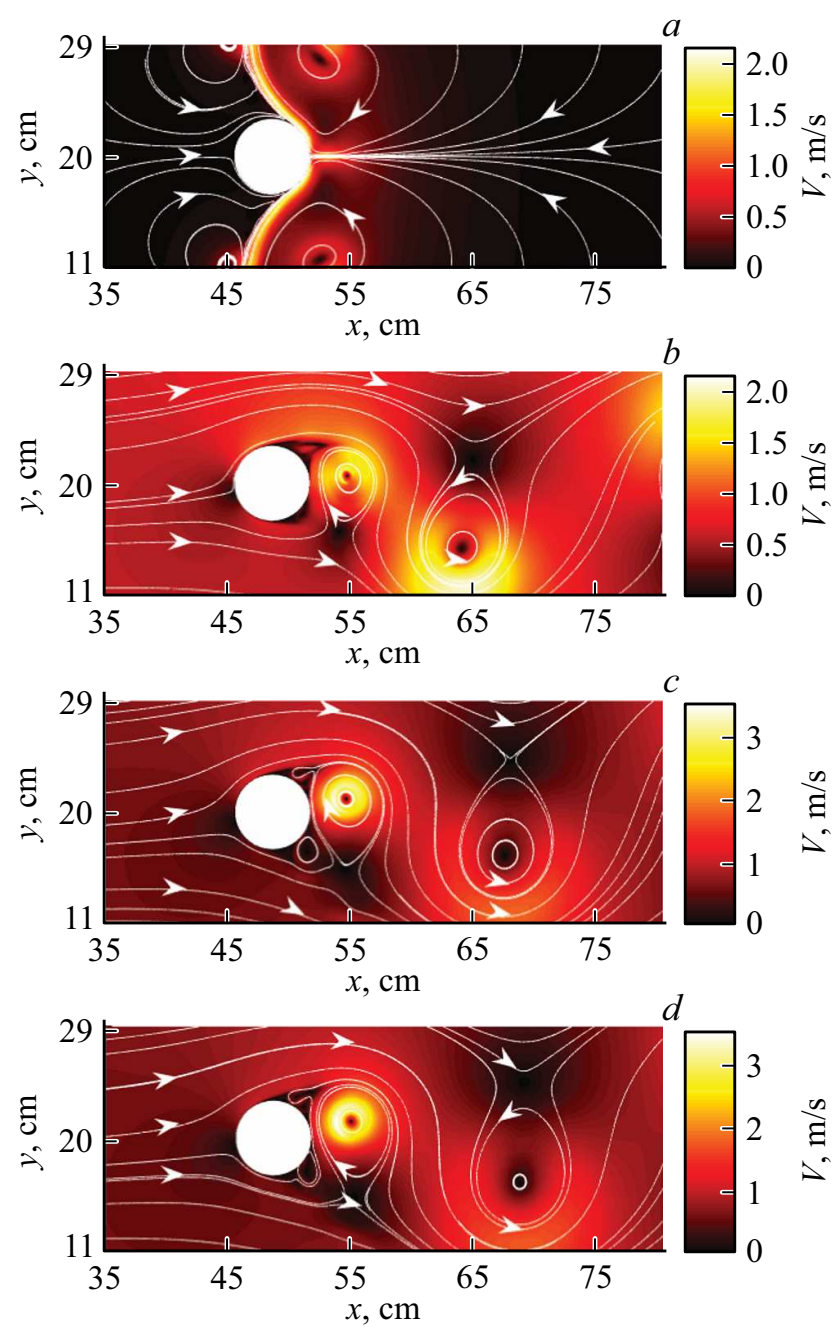

Рис. 4. Распределение модуля скорости $V$ и линии тока для $\theta=180^{\circ}: a-V_{0}=0, U=16.2 \mathrm{kV}, b-V_{0}=0.6 \mathrm{~m} / \mathrm{s}$, $U=16.2 \mathrm{kV}, c-V_{0}=0.6 \mathrm{~m} / \mathrm{s}, U=19.2 \mathrm{kV}, d-V_{0}=0.6 \mathrm{~m} / \mathrm{s}$, $U=21.2 \mathrm{kV}$.
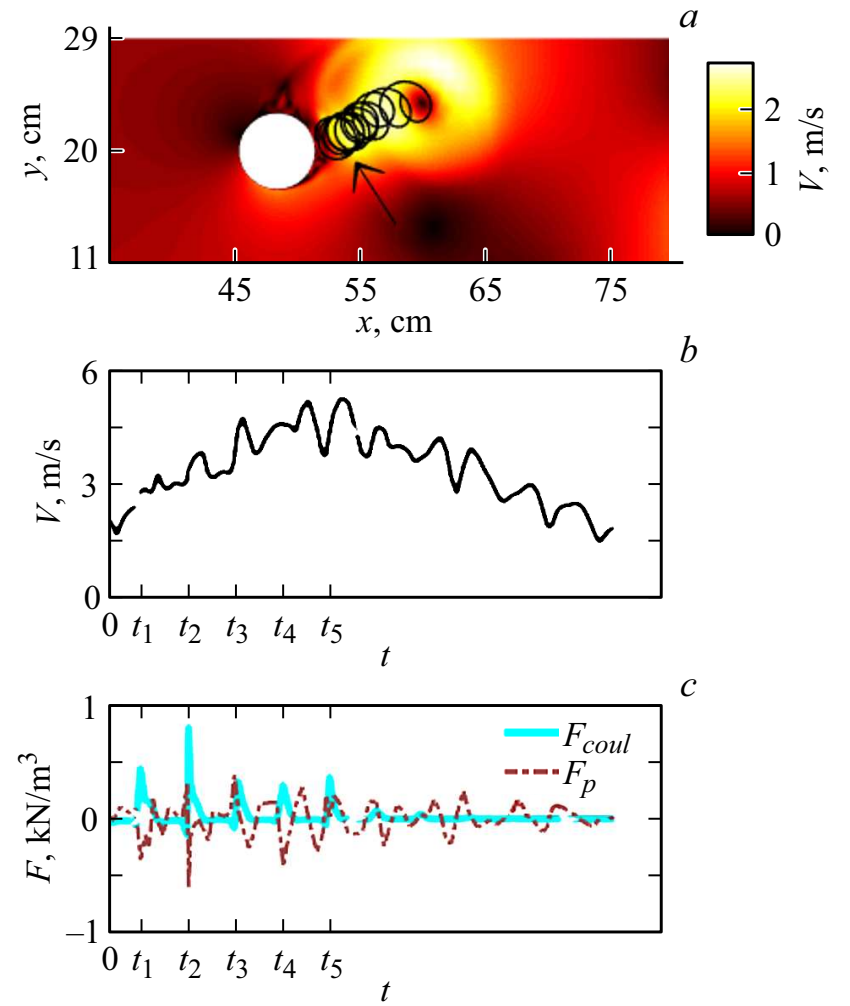

Рис. 5. Влияние сил, действующих на пробный объем газа, на его скорость при движении в вихревом следе, $V_{0}=0.6 \mathrm{~m} / \mathrm{s}, U=24.2 \mathrm{kV}, \theta=180^{\circ}: a-$ спиралевидная траектория пробного объема (стрелкой показано положение электрода), $b-$ изменение скорости пробного объема вдоль траектории, $c$ - изменение проекций сил Кулона $\left(F_{\text {coul }}\right)$ и давления $\left(F_{p}\right)$ на направление движения пробного объема.

Коэффициенты лобового сопротивления $C_{x}$ и подъемной силы $C_{y}$ определяются как:

$$
C_{x}=\frac{2 F_{x}}{\rho V_{0}^{2} A}, C_{y}=\frac{2 F_{y}}{\rho V_{0}^{2} A},
$$

где $F_{x}$ и $F_{y}$ - силы, действующие на цилиндр, по осям $x$ и $y, A$ - миделево сечение цилиндра.

На рис. 6 показаны временные зависимости коэффициентов $C_{x}$ и $C_{y}$. Графики 1 и 2 на рис. 6, $a-$ результат классической дорожки Кармана. Наблюдаются периодические колебания для подъемной силы относительно нуля и для силы сопротивления относительно среднего значения 1.4. Эта величина заметно завышена по сравнению с экспериментальными данными по обтеканию цилиндра $[5,10]$. Такое расхождение можно объяснить не учетом загромождения канала. В работе [11] приводится корректировочная формула, дающая поправочный коэффициент 1.37. Тогда скорректированное значение коэффициента сопротивления $C_{x}=1.0$ становится ближе к экспериментальным значениям 0.9 для чисел Рейнольдса около 2000. Период колебаний подъемной силы $T=0.46$ с, следовательно, число Стру- 

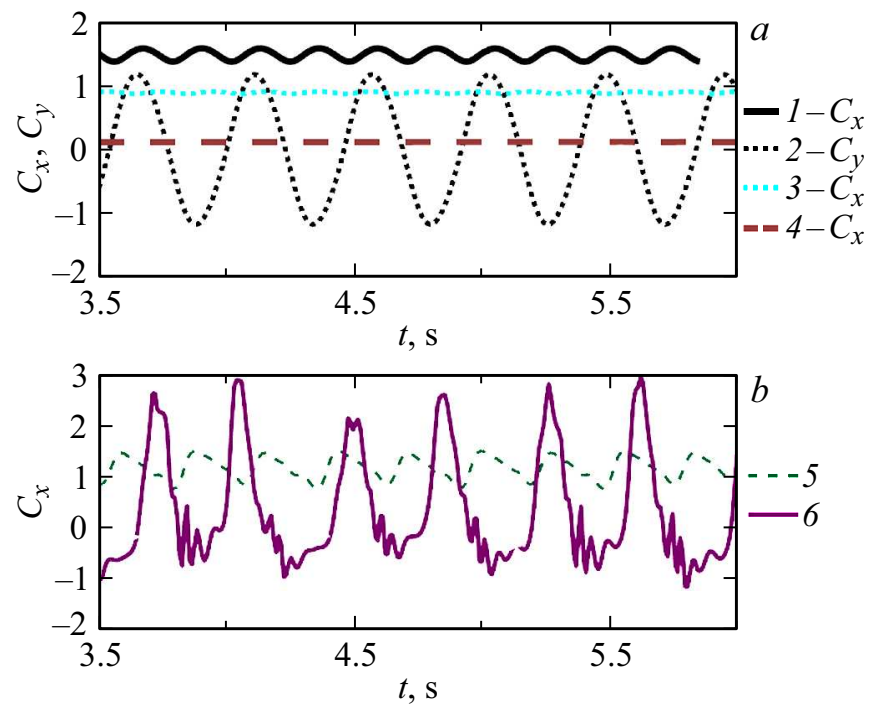

Рис. 6. Зависимость $C_{x}$ и $C_{y}$ от времени для $V_{0}=0.6 \mathrm{~m} / \mathrm{s}: a-$ $\left.\theta= \pm 90^{\circ}: 1,2\right)-U=0,3-U=10.2 \mathrm{kV}, 4-U=16.2 \mathrm{kV}$; $b-\theta=180^{\circ}: 5-U=16.2 \mathrm{kV}, 6-U=26.2 \mathrm{kV}$.

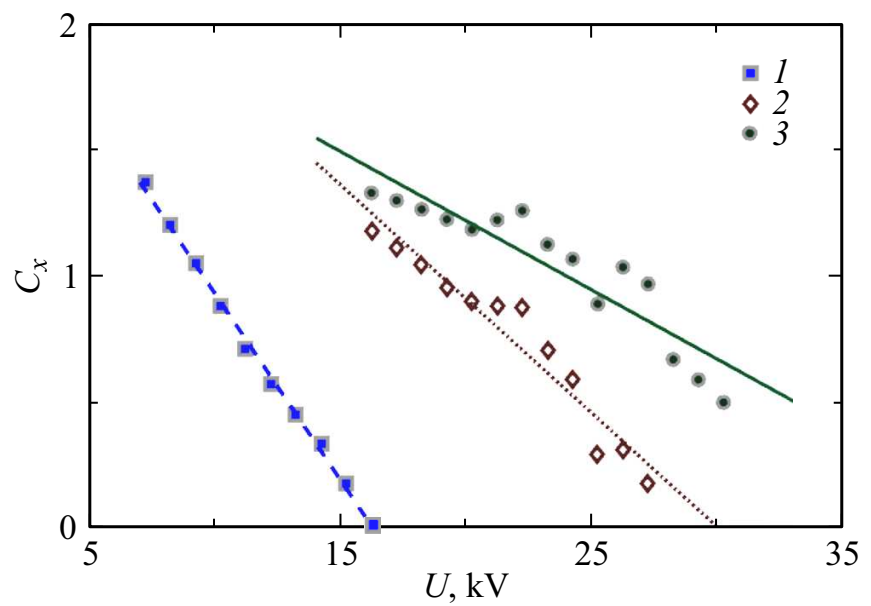

Рис. 7. Зависимость от напряжения среднего по периоду коэффициента лобового сопротивления $C_{x}: 1-V_{0}=0.6 \mathrm{~m} / \mathrm{s}$, $\theta= \pm 90^{\circ}, 2-V_{0}=0.6 \mathrm{~m} / \mathrm{s}, \theta=180^{\circ}, 3-V_{0}=0.8 \mathrm{~m} / \mathrm{s}$, $\theta=180^{\circ}$.

халя, определяемое по формуле (7), равно 0.22:

$$
S t=\frac{2 R}{V_{0} T} .
$$

Экспериментальные значения числа Струхаля для рассматриваемых чисел Рейнольдса равны 0.21. Такое совпадение кажется достаточно хорошим. 5 и 10\% отличие для $S t$ и $C_{x}$ соответственно может объясняться неточным сопоставлением эксперимента и моделирования, например, разными условиями на входе для потока. Также нужно учитывать, что при рассматриваемых числах Рейнольдса течение нельзя считать строго двумерным.

На рис. 6, а графики 3 и 4 соответствуют обтеканию с коронным разрядом при расположении электродов над боковой поверхностью цилиндра $\left(\theta= \pm 90^{\circ}\right)$. При увеличении напряжения до $10.2 \mathrm{kV}$ (график 3) амплитуда колебаний и среднее значение $C_{x}$ существенно уменьшаются. Частота колебаний при этом меняется незначительно. При увеличении напряжения до $16 \mathrm{kV}$ (график 4) колебаний практически нет, а среднее значение коэффициента лобового сопротивления уменьшается на порядок. Этот случай соответствует устойчивому обтеканию, приведенному на рис. $3, d$.

При расположении коронирующего электрода за цилиндром $\left(\theta=180^{\circ}\right)$ картина совершенно иная. Как показано на рис. 4, в этом случае вихри значительно усиливаются ЭВ. С увеличением напряжения амплитуда колебаний силы лобового сопротивления возрастает (рис. 6,b (графики 5,6)), а среднее значение уменьшается. К тому же форма колебаний начинает заметно отличаться от гармонической, и частота колебаний уменьшается (при напряжении $30 \mathrm{kV}$ в 2.5 раза от исходной дорожки Кармана). Форма колебаний и их амплитуда определяется механизмом накопления вихрей и их отрывом от коронирующего электрода. Отрыв вихря приводит к понижению давления за цилиндром и увеличению коэффициента сопротивления (возрастающие участки графика на рис. $6, b)$. Далее начинает расти вихрь на противоположной стороне цилиндра, вихрь усиливается ЭВ и оказывает давление на заднюю часть цилиндра. Это приводит к уменьшению коэффициента сопротивления (убывающие участки графика рис. $6, b$ ). Неустойчивость следа приводит к образованию дополнительных мелких вихрей около поверхности цилиндра (рис. 2,b), которые вызывают дополнительные слабые быстрые колебания силы лобового сопротивления (рис. 6, $b$ ).

На рис. 7 показан график зависимости среднего по периоду коэффициента лобового сопротивления от напряжения на высоковольтном электроде. Как видно из рисунка, спад коэффициента сопротивления значителен для каждого из двух рассматриваемых положений коронирующего электрода. Однако при $\theta= \pm 90^{\circ}$ эффект проявляется при более низких напряжениях (график 1). К тому же в этом случае уменьшение средней величины сопровождается уменышением амплитуды колебания. При $\theta=180^{\circ}$ амплитуда колебаний силы лобового сопротивления растет в связи с формированием крупных вихрей, а ее среднее значение падает. При увеличении скорости входящего потока до $0.8 \mathrm{~m} / \mathrm{s}$ ЭВ значительно слабее изменяет среднюю силу лобового сопротивления (графики 2 и 3). Видно, что для ряда скоростей набегающего потока можно подобрать такое напряжение, при котором ЭВ в зависимости от расположения электродов полностью или в среднем компенсирует воздействие входящего потока. Однако для коронного разряда увеличение напряжения ограничено напряжением пробоя между электродами. 


\section{Заключение}

С помощью компьютерного моделирования было проведено исследование обтекания кругового цилиндра в нестационарном режиме для чисел Рейнольдса 2400 и 3200 с дополнительной объемной электрической силой, созданной коронным разрядом. Расчет показал, что в рассматриваемом диапазоне параметров коронный разряд может заметно повлиять на характер обтекания цилиндра внешним потоком воздуха, сместить положение точек отрыва пограничного слоя и изменить характеристики дорожки Кармана. Степень воздействия существенно зависит от расположения коронирующего электрода.

При расположении коронирующего электрода за цилиндром коронный разряд оказался способен значительно уменьшить частоту образования вихрей по сравнению с дорожкой Кармана без коронного разряда. При этом образовывались вихри больших размеров с большей скоростью вращения. С увеличением напряжения разряда вихри накапливают больший момент импульса, поскольку им мешает оторваться и раскручивает прижимающий их ЭВ. Увеличение времени накопления ведет к уменьшению средней силы лобового сопротивления, но при этом амплитуда колебаний силы увеличивается, так как за накоплением идет отрыв, во время которого сила сопротивления возрастает. При расположении двух коронирующих электродов симметрично сверху и снизу цилиндра картина взаимодействия коронного разряда с натекающим потоком воздуха иная. В этом случае в области коронирующих электродов возникает область разряжения, которая всасывает набегающий поток воздуха, изменяя условия формирования дорожки Кармана. Точка отрыва пограничного слоя смещается вверх по течению, а линии тока основного течения сгущаются в зоне пониженного давления у коронирующих электродов, а затем вновь приближаются к поверхности цилиндра, происходит присоединение оторвавшегося потока. С увеличением напряжения частота образования вихрей не меняется, а размер следа уменьшается. Для исследуемых условий след исчез полностью при напряжении $16.2 \mathrm{kV}$, течение стало стационарным и симметричным. Среднее значение и амплитуда колебаний силы лобового сопротивления падают с увеличением напряжения коронного разряда. Можно подобрать такое напряжение коронного разряда, при котором средняя сила лобового сопротивления уменьшается практически до нуля. Однако с увеличением скорости входящего потока для достижения подобного равновесия нужно увеличивать напряжение, которое ограничено напряжением пробоя для выбранной системы электродов.

\section{Благодарности}

Исследования проведены с использованием оборудования ресурсного центра Научного парка СПбГУ „Вычислительный центр“.

\section{Конфликт интересов}

Авторы заявляют, что у них нет конфликта интересов.

\section{Список литературы}

[1] Vatazhin A.B., Likhter V.A., Ulybyshev Dynamics. 2012. Vol. 47. N 2. P. 206-213. DOI: $10.1134 / \mathrm{S} 0015462812020081$

[2] Moreau E. // J. Phys. D. Appl. Phys. 2007. Vol. 40. P. 605-636. DOI: $10.1088 / 0022-3727 / 40 / 3 / \mathrm{S} 01$

[3] Artana G., Sosa R., Moreau E., Touchard G. // Exp. Fluids 2003. Vol. 35. P. 580-588. DOI: $10.1007 / \mathrm{s} 00348-003-0704-\mathrm{z}$

[4] Hyun K.T., Chun C.H. // Exp. Fluid. 2003. Vol. 35. P. 541-552. DOI: $10.1007 / \mathrm{s} 00348-003-0668-\mathrm{z}$

[5] Жукаускас А., Жюгжди И. Теплоотдача цилиндра в поперечном потоке жидкости. Вильнюс: Мокслас, 1979. $240 \mathrm{c}$.

[6] Deylami H.M., Amanifard N., Hosseininezhad S.S., Dolati F. // Eur. J. Mech. B. Fluid. 2017. Vol. 66. P. 71-80. DOI: $10.1016 /$ j.euromechflu.2017.06.002

[7] Райзер Ю.П. Физика газового разряда. Долгопрудный: Интеллект, 2009. 736 с.

[8] Самусенко А.В., Сафронова Ю.Ф., Стишков Ю.К. // Электронная обработка материалов. 2016. Т. 52. № 5. C. 43-50.

[9] Zhidkova P.S., Samusenko A.V. // Surf. Eng. Appl. Elect. 2016. Vol. 52. N 4. P. 370-379. DOI: 10.3103/S106837551604013X

[10] Roshko A. On the development of turbulent wakes from vortex streets. Washington, NACA Rep. 1191. 1954.

[11] Ota T., Okamoto Y., Yoshikawa H.A // J. Fluid. Eng. 1994. Vol. 116. N 3. P. 414-418. DOI: 10.1115/1.2910292 